\title{
Special Issue on Synapse Assembly, Neural Circuit Development, and Brain Disorders
}

\author{
Jaewon $\mathrm{Ko}^{1}$ and Eunjoon $\mathrm{Kim}^{2,3}$
}

Synapses are specialized junctions between neurons in the brain that transmit and compute neural information, thereby connecting billions of neurons into neural circuits. Neural circuits are currently considered as neural communication hubs and nodes, by virtue of their connecting neurons via various synapse types ${ }^{1}$. Neurons constantly form and eliminate synapses throughout brain development through various cell biological processes, including synapse establishment through axon-dendrite contact $^{2-4}$, synapse formation through the assembly of various synaptic components in both presynaptic and postsynaptic neurons ${ }^{5-7}$, synapse maturation through the recruitment of various synaptic plasticity mechanisms ${ }^{8}$, and synapse elimination through activity-dependent mechanisms that may involve several glial cell types (astrocytes and microglia) $^{9-11}$. All of these processes are likely mediated by specific molecular pathways that confer characteristic synaptic properties, including neurotransmitter type, neurotransmitter-release probability, plasticity, postsynaptic receptor composition, and synaptic adhesion ${ }^{1}$. Neuroscientists have approached numerous questions related to various synaptic properties using molecular and cellular neuroscience tools and have contributed to the establishment of Systems Neuroscience through the development of new technologies for manipulating and visualizing neural circuits ${ }^{12}$. The goal of this special issue in Experimental and Molecular Medicine (EMM), entitled 'Synapse Assembly, Neural Circuit Development and Brain Disorders', is to highlight the significance of molecular and cellular neuroscience studies to encourage readers to think about ways in which

\footnotetext{
Correspondence: Jaewon Ko (jaewonko@dgist.ac.kr)

'Department of Brain \& Cognitive Sciences, Daegu Gyeongbuk Institute of

Science and Technology (DGIST), Daegu 42988, Korea

${ }^{2}$ Center for Synaptic Brain Dysfunctions, Institute of Basic Science (IBS), Daejeon

34141, Korea
}

Full list of author information is available at the end of the article brain structure and function can be understood in a more comprehensive manner.

In this special issue, we present a collection of review articles that provide keen insights into current trending topics in the realm of molecular and cellular neuroscience. In the first article, de Wit and colleagues introduce a recently emerging class of synaptic adhesion molecules that likely shape various aspects of synapse development. Specifically, they highlight the roles of leucine-rich repeat-containing cell surface proteins in mediating synapse formation and transmission in various types of synapses and neural circuits. Both Han and colleagues and Chang and colleagues pinpoint an imbalanced excitation-to-inhibition (E/I) ratio as a key pathophysiological mechanism underlying brain disorders such as mania and mental disorders, focusing on recent rodent studies. They discuss the roles of various synaptic proteins, all of which are essential for a subset of synapse developmental processes, and provide suggestions regarding how some of these synaptic proteins and the processes in which they are involved could be considered therapeutic targets for brain disorders. Um and colleagues review the roles of immediate early genes (IEGs), which are workhorses in the regulation of synaptic activitydependent brain development, focusing on three prominent IEGs-Arc, Npas4 and Homer1a-that have also been implicated in certain brain disorders. Krueger-Burg and colleagues discuss the synaptic and circuit mechanisms underlying fear and anxiety, focusing on amygdalar GABAergic interneurons. They further highlight the roles of GABA receptors and a subset of key inhibitory synapse organizers that shape inhibitory synaptic transmission within the amygdalar neural circuits. Lastly, Choi discusses the molecular and cellular mechanisms underlying primary sensory cortical development. Intriguingly, he underscores the significance of a critical period of plasticity in the development of the sensory cortex and 
describes some of the key molecular mechanisms that drive its structural and functional organization.

We hope that the review articles in this Special Issue of EMM are useful and can bring the varied expertize of readers to molecular and cellular neuroscience fields, since such broad input is important in continuing to make strides in revealing key discoveries that we believe are critical for progress in curing brain disorders. On a final note, we would like to express our appreciation for the efforts of all contributors in assembling their pieces for this special issue.

\section{Acknowledgements}

This work was supported by grants from the National Research Foundation of Korea (NRF) funded by the Ministry of Science and ICT (2016R1A2B200682 to J. K.) and the Institute for Basic Science (IBS-R002-D1 to E.K.).

\section{Author details}

'Department of Brain \& Cognitive Sciences, Daegu Gyeongbuk Institute of Science and Technology (DGIST), Daegu 42988, Korea. ${ }^{2}$ Center for Synaptic Brain Dysfunctions, Institute of Basic Science (IBS), Daejeon 34141, Korea.

${ }^{3}$ Department of Biological Sciences, Korea Advanced Institute of Science and Technology (KAIST), Daejeon 34141, Korea

\section{Conflict of interest}

The authors declare that they have no conflict of interest.

\section{Publisher's note}

Springer Nature remains neutral with regard to jurisdictional claims in published maps and institutional affiliations
Received: 5 December 2017 Accepted: 6 December 2017

Published online: 9 April 2018

References

1. Südhof, T. C. Synaptic neurexin complexes: a molecular code for the logic of neural circuits. Cell 171, 745-769 (2017).

2. Um, J. W. \& Ko, J. LAR-RPTPs: synaptic adhesion molecules that shape synapse development. Trends Cell. Biol. 23, 465-475 (2013).

3. de Wit, J. \& Ghosh, A. Specification of synaptic connectivity by cell surface interactions. Nat. Rev. Neurosci. 17, 22-35 (2016).

4. Takahashi, H. \& Craig, A. M. Protein tyrosine phosphatases PTPdelta, PTPsigma, and LAR: presynaptic hubs for synapse organization. Trends Neurosci. 36, 534-552 (2013).

5. Kim, E. \& Sheng, M. PDZ domain proteins of synapses. Nat. Rev. Neurosci. 5 771-781 (2004).

6. Petzoldt, A. G., Lutzkendorf, J. \& Sigrist, S. J. Mechanisms controlling assembly and plasticity of presynaptic active zone scaffolds. Curr. Opin. Neurobiol. 39, 69-76 (2016).

7. Sheng, M. \& Kim, E. The postsynaptic organization of synapses. Cold Spring Harb. Perspect. Biol. 3, pii: a005678 (2011).

8. Nicoll, R. A. A brief history of long-term potentiation. Neuron 93, 281-290 (2017).

9. Allen, N. J. \& Eroglu, C. Cell biology of astrocyte-synapse interactions. Neuron 96, 697-708 (2017)

10. Chung, W. S., Allen, N. J. \& Eroglu, C. Astrocytes control synapse formation, function, and elimination. Cold Spring Harb. Perspect. Biol. 7, a020370 (2015).

11. Eroglu, C. \& Barres, B. A. Regulation of synaptic connectivity by glia. Nature $\mathbf{4 6 8}$ 222-231 (2010)

12. Südhof, T. C. Molecular neuroscience in the 21st Century: a personal perspective. Neuron 96, 536-541 (2017). 